\title{
Colorectal Cancer - Management of Metastatic Disease
}

\author{
Paul H Sugarbaker
}

Medical Director, Center for Gastrointestinal Malignancies at MedStar Washington Hospital Center, Washington, DC and Chief,

Program in Peritoneal Surface Oncology at MedStar Washington Cancer Institute, Washington, DC, US

\begin{abstract}
Metastases from colon cancer occur to the regional lymph nodes, the liver and the peritoneal surfaces. Rectal cancer may disseminate to these sites and also to the lungs. These metastases may occur synchronously with the detection of primary disease or metachronously in follow-up. The timing of the metastatic process is important in terms of treatment possibilities. Each anatomical site for metastatic disease has the potential for an individualised management strategy. Systemic chemotherapy as an adequate management plan for all sites of colorectal metastatic disease is not compatible with a high standard of care. Formulation of an optimal plan combining surgery with regional and systemic chemotherapy is a necessary function of the multidisciplinary team.
\end{abstract}

\section{Keywords}

Lymph node metastases, liver metastases, peritoneal metastases, peritoneal carcinomatosis, lung metastases, hyperthermic intraperitoneal chemotherapy (HIPEC), early post-operative intraperitoneal chemotherapy (EPIC)

Disclosure: The author has no conflicts of interest to declare.

Received: 17 October 2013 Accepted: 29 October 2013 Citation: European Oncology \& Haematology, 2013;9(2):100-4 DOI: 10.17925/EOH.2013.09.2.100

Correspondence: Paul H Sugarbaker, 106 Irving Street, NW, Suite 3900, Washington, DC 20010, US. E: Paul.Sugarbaker@medstar.net

The management of colorectal cancer has continued to evolve over approximately one century. Without a doubt the most effective management strategy to combat this disease is prevention. This involves the identification of high-risk groups, dietary changes and dietary supplements. ${ }^{1}$ The next most effective management strategy is screening for disease to confirm a diagnosis of colon or rectal cancer in its early natural history. The use of the haemoccult test on a regular basis has been proven effective. ${ }^{2}$ Better yet, for screening is complete colonoscopy. ${ }^{3}$ In symptomatic patients, the surgical management strategies have been well defined for both colon cancer and for rectal cancer. The surgery must provide a complete clearance of the primary cancer and its lymph node groups at risk for metastatic disease. The resection must be accomplished with perfect containment of the process. ${ }^{4,5} \mathrm{~A}$ tragic modern-day surgical reality continues in that a patient may enter the operating room with a contained process and leave with disseminated disease. Iatrogenic cancer dissemination results from trauma to the surgical specimen so that cancer cells are not contained and are lost from the specimen into the resection site or free peritoneal cavity. ${ }^{6}$ The long-term result is local recurrence and peritoneal metastases. This can occur with open colorectal surgery or with laparoscopic resection.

This manuscript is not a commentary on the majority of patients who have an uncomplicated colorectal cancer resection and a favourable prognosis. It concerns the approximately $30 \%$ of patients who have advanced disease at the time of presentation and the $50 \%$ of patients who months or years after resection are found to have progressive disease as a result of treatment failure of primary disease. The focus is on local recurrence and metastases from colon and rectal cancer. ${ }^{\text {? }}$

\section{Lymph Nodal Metastases from Colorectal Cancer}

In the past, extensive lymphadenectomy as part of a colorectal cancer resection was thought by many surgeons to be unnecessary. The rationale was that patients with metastases to the intermediate or para-aortic nodes could not survive even if these nodes were resected as part of the primary colorectal cancer surgical intervention. Recent data suggests that this retreat to a 'conservative resection' was not indicated. Rather, data now shows that one should perform a wide resection of lymph nodes to the superior mesenteric vessels with a right colon cancer. With a left-sided malignancy, nodes should be resected to the origin of the inferior mesenteric artery.

Swanson and colleagues reported on the survival of 35,787 prospectively collected cases of T3NO colon cancers that were surgically treated and pathologically reported from 1985-91. T3 cancers would be expected to be at a higher risk for lymph nodal metastases as compared to T1 or T2 lesions and therefore, adequate lymphadenectomy would show a greater benefit in this subgroup of patients. The five-year survival of T3NOMO colon cancer patients varied from $64 \%$ if one or two lymph nodes were examined to $86 \%$ if greater than 25 lymph nodes were examined. Three strata of resected lymph nodes (1-7, 8-12 and greater than 13) resulted in significantly different five-year survival rates. The authors conclude that the prognosis of T3NO colon cancer patients is dependent on the number of lymph nodes examined and suggest a minimum of 13 lymph nodes to be resected. ${ }^{8}$

Le Voyer and colleagues reported on survival from an intergroup trial, INT-0089. In 3,411 assessable patients, 648 had no evidence of lymph node metastases. Multivariate analyses were performed on both 
the node positive and node negative groups separately to ascertain the effect of extent of lymph node resection on survival. As might be expected, survival decreased with increasing number of lymph nodes involved $(p=0.0001)$. After controlling for the number of nodes involved, survival increased as more nodes were analysed ( $p=0.0001)$. Even when no nodes were involved, overall survival and cause-specific survival improved as more nodes were available for analysis ( $p=0.0005$ and $p=0.007$, respectively). The authors conclude that the number of lymph nodes resected and available for analysis for staging colon cancer is itself a prognostic variable on outcome. ${ }^{9}$

West and colleagues investigated prognostic implications of the plane of surgical resection of colonic cancer. The complete mesocolic excision with central vascular ligation produced a survival of greater than $89 \%$. There was a greater yield of lymph nodes in 49 specimens from Erlangen, Germany as compared to 40 standard specimens from Leeds, UK; a lymph node yield of 30 in Erlangen was compared to 18 in Leeds $(p<0.0001)$. West and colleagues conclude that the plane of colon cancer resection and the extent of lymphadenectomy are important in optimal surgical technique. The improved containment of a mesocolic resection combined with the associated larger number of lymph nodes removed was suggested as the explanation for improved survival rates reported in Erlangen. ${ }^{4}$

\section{Hepatic Metastases from Colorectal Cancer}

Due to the pioneering efforts of Wilson and Adson, Foster and Berman, and Hughes and colleagues, the benefits that occur with the resection of liver metastases from metastatic colorectal cancer have been established as a standard of practice. ${ }^{10-12}$ This change in the established guidelines has occurred despite the fact that there has no verification of the -evidence in terms of a Phase III randomised controlled study. ${ }^{13}$ Overall, the five-year survival following R-0 hepatic resection for colorectal metastases is $30-50 \%$. There may be some improvement in this statistic as a result of repeat hepatic resections that have been shown to be successful. ${ }^{14}$ In some patients with liver metastases that are unresectable because of a large extent of disease, systemic chemotherapy can downsize the liver metastases so that an $\mathrm{R}-0$ resection is possible. The survival of this group of patients receiving neoadjuvant chemotherapy is nearly identical to patients having surgery as an initial treatment. ${ }^{15}$

Patient-related factors associated with poor outcome include elevation in serum carcinoembryonic antigen (CEA), positive lymph node status of the primary tumour, lymph nodes present in the regional peri-portal lymph nodes and disease-free interval of less than one year. Factors in respect to liver metastases that carry a poor prognosis are increasing number of lesions, increasing size of the largest lesion, bilobar distribution and percentage of hepatic replacement by cancer. Also, technical factors such as a positive or close margins of resection carry a reduced prognosis. ${ }^{12,16}$

The technology used to accomplish an R-0 resection of the liver metastases may vary greatly between institutions. The anatomical location, size and number of metastases may also favour one method of treatment over another. With multiple metastases limited to either the right or left lobe, a right or left hepatectomy is indicated. Also a lesion greater than $5 \mathrm{~cm}$ usually requires hemihepatectomy. However, segmental resection with clear margins may be indicated if an R-0 resection is possible. Radiofrequency or microwave ablation is an option if metastases are less than $5 \mathrm{~cm}$ and are not immediately adjacent to a major bile duct or major vascular structure. ${ }^{17,18}$ Indications for a cryogenic ablation are similar as for heat ablation. ${ }^{19} \mathrm{~A}$ frequent indication for an ablative procedure in combination with resection is one or two metastases deep in the residual liver after a hemihepatectomy.

Recently, percutaneous ablation procedures have been reported..$^{20}$ Results in properly selected patients seem to be acceptable. Laparoscopic liver resection has also been reported and associated with a reduced hospital stay. ${ }^{21}$

\section{Management of Peritoneal Metastases}

Survival benefits for peritoneal metastases from colon and rectal cancer using cytoreductive surgery and peri-operative chemotherapy began to appear in publications in the 1990s. In 1995, Sugarbaker and Jablonski showed a three-year survival of $35 \%$ in patients with peritoneal metastases from colon cancer treated with cytoreductive surgery plus early post-operative intraperitoneal mitomycin C and fluorouracil.22 Shen and colleagues accumulated patients between 1991 and 2002. Seventy-seven non-appendiceal colorectal cancer patients underwent the combined treatment. They concluded that one-third of patients with complete resection have long-term survival and that systemic chemotherapy did not contribute to the control of peritoneal metastases. ${ }^{23}$ In 2003, Verwaal and colleagues from Amsterdam published a three-year projected survival of $38 \%$ in 54 patients treated by cytoreductive surgery and hyperthermic intraperitoneal mitomycin $\mathrm{C}$ with adjuvant systemic 5 -fluorouracil. ${ }^{24}$ These studies, performed in the absence of modern colorectal cancer chemotherapy (oxaliplatin and irinotecan), document the efficacy of cytoreductive surgery and peri-operative chemotherapy to rescue approximately one-third of patients with peritoneal metastases. Undoubtedly, patient selection had an important role in achieving these long-term survivals. However, the uniformly fatal outcome of peritoneal metastases no matter the selection factors, establishes the ultimate importance of these early reports.

Since 2003, multiple publications confirming the efficacy of the combination of cytoreductive surgery and peri-operative chemotherapy to benefit patients with colorectal peritoneal metastases have been published. Glehen, in a multi-institutional retrospective study of 506 patients from 28 institutions, reported an overall median survival of 19.2 months in patients with peritoneal metastases from colorectal cancer treated with the combined approach. Of great importance, patients in whom the cytoreductive surgery was complete had a median survival of 32.4 months as compared to 8.4 months in patients in whom cytoreduction was not completed $(p<0.001)$. The morbidity was $22.9 \%$ and mortality $4 \%$. The authors conclude that the therapeutic approach of combining cytoreductive surgery with peri-operative intraperitoneal chemotherapy achieved long-term survival in a selected group of patients with peritoneal metastases of colorectal origin with acceptable morbidity and mortality. The complete cytoreduction was the most important prognostic indicator. ${ }^{25}$

Elias and colleagues reported on colorectal peritoneal metastases in a retrospective analysis of 523 patients from 23 French-speaking centres. The overall median survival was 30.1 months and the five-year overall survival was $27 \%$. Eighty-four per cent of the patients had a complete cytoreduction with a median survival of 33 months. This group concluded that cytoreductive surgery and peri-operative chemotherapy is now considered the gold standard in the French guidelines for management of peritoneal metastases. ${ }^{26}$ Similarly, verwaal reported a long-term 
Dutch multicentre data analysis. The survival of 562 patients at 10 years was $37 \%{ }^{27}$

At the top of the list regarding evidence-based medicine for treatment of peritoneal metastases is the Phase III study reported by Verwaal and colleagues in 2003. This landmark study compared 105 colorectal peritoneal metastases patients who were randomly assigned to receive either standard treatment with systemic 5-fluorouracil and leucovorin or an aggressive cytoreductive surgery with peri-operative chemotherapy using hyperthermic mitomycin C. The patients in the 'experimental therapy' arm also received systemic 5-fluorouracil chemotherapy. After a median follow-up of 21.6 months, the median survival was 12.6 months with systemic chemotherapy and 22.3 months with cytoreduction and peri-operative chemotherapy ( $p=0.032)$. These authors reported that a complete cytoreduction and a limited extent of disease were important determinants of benefit. ${ }^{28}$ The durability of the benefit of cytoreductive surgery and peri-operative chemotherapy was confirmed in a follow-up manuscript in 2008.29

Recently, these benefits have been called into question by Ryan. He has questioned the relevance of cytoreductive surgery and peri-operative chemotherapy now that oxaliplatin, irinotecan and molecular agents are available. He contends that the benefits of systemic chemotherapy alone are so great that cytoreduction plus peri-operative chemotherapy is no longer indicated. ${ }^{30}$ However, current data confirms that for limited extent of peritoneal metastases, a multidisciplinary approach using the best surgical and best chemotherapy treatments is preferable. Franko and colleagues presented data to show that these two options work best when used together. They showed that the median survival was longer in patients treated by modern systemic chemotherapy when cytoreductive surgery and hyperthermic intraperitoneal chemotherapy (HIPEC) was added to the clinical pathway. ${ }^{31}$ Currently, standard of care, until more data becomes available, indicates that patients with peritoneal metastases from colorectal cancer have the right to be informed of a possible curative treatment option. It is the oncologist's obligation to provide the relevant information in a timely fashion. ${ }^{32}$

\section{Lung Metastases From Colorectal Cancer}

Thoracotomy to remove metastatic colorectal cancer has been advocated for selected patients since the early 1980s. Lung metastases, in the absence of metastases at other sites, occur in a minority of colorectal cancer patients. In one series of 1,578 patients who underwent a potentially curative resection of colorectal cancer, 137 (8.7 \%) developed lung metastases and only 16 (1\%) were candidates for thoracotomy. Also, the prognosis of lung metastases from colon cancer and lung metastases from rectal cancer are different. Eleven and a half per cent were resected from rectal cancer and only $3.6 \%$ from colon cancer patients. ${ }^{33}$ Currently, favourable factors for resection include solitary metastases, carcinoembryonic antigen (CEA) less than $5 \mathrm{ng} / \mathrm{ml}$ and a disease-free interval of greater than three years. ${ }^{34}$

\section{Technology of Pulmonary Metastases Resection or Ablation}

Originally, lung metastases were resected through a lateral thoracostomy incision. In selected patients with bilateral pulmonary metastases a bilateral thoracotomy may facilitate the resection by eradication of disease from both lungs with a single intervention. ${ }^{35}$

Thorascopic intervention may identify and then resect the pulmonary metastases in the absence of a thoracotomy. The lesion should be three centimetres or less in size, well identified on CT and away from major vessels or bronchi. ${ }^{36}$

Recently, percutaneous ablation of lung nodules has been reported. ${ }^{37}$ This technology in properly selected patients is reported to be well tolerated and effective in the long-term follow-up. Where the expertise is available, the percutaneous ablation may be the treatment of choice in selected patients.

\section{Multiple Sites of Colorectal Metastases Synchronous Metastases}

Frequently, patients with simultaneous liver metastases and lung metastases or liver metastases and peritoneal metastases should be considered for treatment. In general, the 'Elias Rule of Fives' can be applied in this situation. If the patient can be made clinically disease-free and there are five or less sites of metastases, then the attempt at resection should occur. The clinician needs to realise that the morbidity and mortality from combined metastasectomy in liver, lung, or peritoneal space will be increased. Also, a full $30 \%$ of patients will be brought to the operating room for an exploratory laparotomy through a long abdominal incision and complete resection found to be impossible..$^{38}$

\section{Metachronous Metastases}

Patients who present with metachronous metastases present less of problem. The benefits seem to be worth the risk if an $\mathrm{R}-0$ resection of all sites of metastatic disease is possible. Resection of liver metastases after previous lung metastases has been reported ${ }^{39}$ as well as resection of pulmonary metastases after liver metastases sequentially with R-0 resections possible. ${ }^{40}$ Also the use of cytoreductive surgery and hyperthermic peri-operative chemotherapy to treat peritoneal metastases before or after resection of liver metastases is common. However, the occurrence of more than one anatomical site of metastatic disease is associated with a reduced prognosis. ${ }^{41}$ Palliative as well as curative benefits may occur.

\section{Metastases from Metastases}

Frequently, surgical judgments regarding the timing of surgical resection of metastatic disease is not obvious to the clinician. Certainly, the surgeon does not want to proceed with a resection that carries moderate to high morbidity and mortality if in the near future metastatic disease at several other anatomical sites becomes evident. However, the 'watch-and-wait' management strategy carries with it the danger of a larger extent of disease and the possibility for additional sites of metastatic disease. The concept of metastases from metastases is a phenomenon that has been reportedly documented in colorectal cancer patients. August and colleagues reviewed their records of 81 patients who had liver resection for colorectal metastases. In seven patients $(9 \%$ ) at the time of surgery, lymph node metastases within the lymphatic drainage of the liver were documented. All seven of these patients had their extrahepatic lymphatic disease limited to nodes draining the liver, implicating lymphatic dissemination of the hepatic metastases as the mechanism of cancer spread. ${ }^{42}$ Unfortunately, in this situation the likelihood of long-term benefit from a liver resection is reduced. ${ }^{43}$ August and colleagues suggested that a need for frequent and thorough follow-up of patients following resection of primary colorectal cancer is indicated and that an urgency in the definitive management (surgical resection) of liver metastases should occur if possible.

Eveno and colleagues studied the pattern of relapse in women with colorectal peritoneal carcinomatosis that showed ovarian 
metastases. One hundred and five women underwent cytoreductive surgery combined with hyperthermic intra-operative chemotherapy. Sixty-two of the 105 (60\%) had ovarian metastases. The median overall survival of the two groups of women with or without ovarian metastases was not statistically different at 36 and 40 months, respectively. However, the pattern of relapse was different in the two groups. There were 19 patients who had retroperitoneal lymph nodal relapse and 18 of these 19 patients had ovarian metastases. The only predictive factor for retroperitoneal relapse was a history of ovarian metastases $(p=0.0012)$. The authors conclude that retroperitoneal lymph node recurrence was linked to ovarian metastases originating from the colorectal malignancy. Early intervention or an intervention combined with systematic lymphadenectomy was suggested as a treatment option for this group of patients. ${ }^{44}$ Evers and Verwaal showed that the disease-free survival after a cytoreductive surgery plus HIPEC procedure for colorectal or appendiceal peritoneal metastases was significantly lower in women with ovarian metastases. These data may also suggest that retroperitoneal metastases are a sequelae of peritoneal metastases being present within the ovaries. ${ }^{45}$

In patients with resectable lung metastases from primary colorectal cancer, Okumura and colleagues reported that approximately one-third had mediastinal lymph node involvement documented at the time of thoracotomy. ${ }^{46}$ Yedibela and colleagues documented mediastinal involvement in $5 \%$ of patients. ${ }^{47}$ Documentation of hilar metastases from lung metastases indicates a reduced prognosis with resection of pulmonary metastases. Inoue reported a five-year survival of $50 \%$ in patients with negative mediastinal or hilar lymph nodes as compared to $14 \%$ with positive lymph nodes. ${ }^{48}$

\section{Locally Recurrent Rectal Cancer}

Rectal cancer may progress to an advanced stage with little or no symptoms. The large calibre of the mid- and lower rectum can result in an absence of obstructive symptoms. Blood per rectum may be attributed to haemorrhoids and fail to cause the patient to seek medical assistance. Even with pre-operative chemoradiation therapy, T3 and T4 rectal cancer may result in locally recurrent disease. ${ }^{49,50}$

The anatomical location of the recurrence is an important determinant of treatment options. ${ }^{50,51}$ Lateral extension after prior total mesorectal excision is seldom resectable with an adequate margin of resection. Intra-operative radiation therapy may be added to the surgery in selected patients. ${ }^{52}$ If the recurrence is anterior in the female patient, a vaginectomy is usually required. With posterior extension partial sacrectomy may result in negative margins. In nearly all patients with a prior low anterior resection, an abdomino-perineal resection of the residual rectum will be necessary. Long-term survival is observed in only approximately $10 \%$ of these patients. ${ }^{50}$

\section{Brain Metastases}

Metastases to the brain are important in that they are often the final site of metastases with successful control of metastatic disease at several other anatomical sites. For example, patients with successfully treated liver metastases and lung metastases may finally succumb to disease spread to the brain. Also, brain metastases are important in that they show the immense significance of the anatomical location of the dissemination in terms of prognosis. Patients with brain metastases rarely live more than several months after diagnosis. Metastases to this site rapidly cause the demise of the patient. In contrast, a much larger metastasis to the liver is usually not symptomatic, may be successfully removed surgically and has an immensely different prognosis. The difference in prognosis of metastases located within the liver versus metastases located within the peritoneal space has already been demonstrated. ${ }^{53}$

\section{Summary \\ Metastases at Different Anatomic Sites Require Different Management Strategies}

In this review, the great differences in the natural history of colorectal metastases to different anatomical sites has been emphasised. The manuscript would have as one of its major goals a clear demonstration that the management of metastatic disease from colorectal cancer must be an individualised plan. The use of systemic chemotherapy alone as an adequate treatment for all sites of colorectal metastatic disease is not a reasonable management strategy. The treatments must be individualised and utilise all modalities including surgery, radiotherapy, systemic chemotherapy and local-regional chemotherapy.

\section{Knowledgeable Management During Follow-up}

Patients who must undergo treatment for colon and rectal cancer require a knowledgeable assessment of their disease process, not only to determine prognosis, but also to predict likely sites for disease progression. Some patients, for example those in whom peritoneal seeding was documented at the time of the primary colorectal cancer resection, are recommended for second-look surgery combined with hyperthermic peri-operative chemotherapy. ${ }^{54}$ All patients with stage II, III, or IV disease require meticulous follow-up in order to identify progression of disease and then evaluation for additional interventions that may result in prolongation of a high-quality existence. Progression of lymph nodal metastases, liver metastases, peritoneal metastases, lung metastases and locally recurrent disease have treatment options for palliation and, in selected patients, for cure. The treatments with longterm benefit frequently combine an $\mathrm{R}-0$ resection with local-regional and systemic chemotherapy.
1. Willet WC, Stampfer MJ, Colditz GA, et al., Relation of meat, fat, and fiber intake to the risk of colon cancer in a progressive study among women, $N$ Eng/ I Med, 1990;323:1664-72.

2. Mandel $\mathrm{JS}$, Bond $\mathrm{JH}$, Church $T R$, et al., Reducing mortality from colorectal cancer by screening for fecal occult blood, $N$ Engl $J$ Med, 1993:328:1365-71.

3. Lieberman DA, Weiss DG, Bond $\mathrm{JH}$, et al., Use of colonoscopy to screen asymptomatic adults for colorectal cancer, N Eng/ to screen asymptomat 2000;343:162-8.

4. West NP, Hohenberger W, Weber K, et al., Complete mesocolic excision with central vascular ligation produces an oncologically superior specimen compared with standard surgery for carcinoma of the colon, I Clin Oncol, 2010;28(2):272-8.

5. Quirke P, Durdey P, Dixon M, Williams N, Local recurrence of rectal adenocarcinoma due to inadequate surgical resection: histopathological study of lateral tumour spread and surgical excision, Lancet, 1986;2:996-9.

6. Sugarbaker PH, Successful management of microscopic residual disease in large bowel cancer, Cancer Chemother Pharmacol, 1999;43(Suppl.):S15-25.

Sugarbaker $\mathrm{PH}$, Colorectal cancer metastases, a surgical perspective, surg Oncol Clin N Am, 2013:22(2):289-98.

( Swanson RS, Compton CC Stewart AK, Bland KL The prognosis of T3NO colon, ancer is dependent on the number pof Le Voyer TE, Sigurdson ER, Hanlon AL, et al, Colon cancer survival is associated with increasing number of lymph nodes analyzed: a secondary survey of intergroup trial INT-0089, J analyzed: a secondary survey of
Clin Oncol, 2003;21(15):2912-9.

10. Wilson SM, Adson MA, Surgical treatment of hepatic metastases from colorectal cancers, Arch surg, 1976;111:330-4.

11. Foster JH, Berman MM, Solid liver tumors, WB Saunders: Philadelphia, 1977.

12. Hughes KS, Simon RM, Songhorabodi S, et al., Resection of the liver for colorectal carcinoma metastases: A multi institutional study of indications for resection, Surgery, $1987 ; 103: 278-88$
13. Kaido $\mathrm{T}$, Verification of evidence in surgical treatment for colorectal liver metastases, Hepato-Gastroenterology, 2008;55:378-80.

14. Fernandez-Trigo V, Shamsa F, Sugarbaker PH, et al., Repeat liver resections from colorectal metastasis, Surgery, 1995;117:296-304.

15. Adam R, Delvart V, Pascal $G$, et al., Rescue surgery for unresectable colorectal liver metastases downstaged by chemotherapy: a model to predict long-term survival, Ann Surg, 2004;240:644-57.

16. Sugarbaker $\mathrm{PH}$, Surgical management of primary and metastatic cancer of the liver, In: Shiff $E$ (Ed.), Diseases of the Liver, JB Lippincott: Philadelphia, 1993:1297-319.

17. Abdalla EK, Vauthey JN, Ellis LM, et al., Recurrence and outcomes following hepatic resection, radiofrequency ablation, and combined resection/ablation for colorectal liver metastases, Ann Surg, 2004;239(6):818-25.

18. Groeschl RT, Pilgrim $\mathrm{CH}$, Hanna EM, et al., Microwave ablation for hepatic malignancies: A multidisciplinary analysis, Ann Surg, 2013; [Epub ahead of print] 
19. Joosten J, Jager G, Oyen W, et al., Cryosurgery and radiofrequency ablation for unresectable colorectal live metastases, Eur J Surg Oncol, 2005;31(10):1152-9.

20. Solbiati L, Livraghi T, Goldberg SN, et al., Percutaneous radiofrequency ablation of hepatic metastases from colorectal cancer: long-term results in 117 patients, Radiology, 2001;221(1):159-66.

21. Buell JF, Cherqui D, Geller DA, et al., The international position on laparoscopic liver surgery: The Louisville Statement, 2008, Ann Surg, 2009;250:825-30.

22. Sugarbaker PH, Jablonski KA, Prognostic features of 51 colorectal and 130 appendiceal cancer patients with peritoneal carcinomatosis treated by cytoreductive surgery and intraperitoneal chemotherapy Ann Surg 1995;221(2):124-32.

23. Shen P, Hawksworth J, Lovato J, et al., Cytoreductive surgery and intraperitoneal hyperthermic chemotherapy with mitomycin C for peritoneal carcinomatosis from nonappendiceal colorectal carcinoma, Ann Surg Oncol, 2004;11(2):178-86

24. Verwaal VJ, van Ruth S, Witkamp A, et al., Long-term survival of peritoneal carcinomatosis of colorectal origin, Ann Surg Oncol, 2005;12(1):65-71.

25. Glehen O, Kwiatkowski F, Sugarbaker PH, et al., Cytoreductive surgery combined with perioperative intraperitoneal chemotherapy for the management of peritoneal carcinomatosis from colorectal cancer: A multi-institutional study, J Clin Oncol, 2004:22(16):3284-92.

26. Elias D, Gilly F Boutitie F et al, Peritoneal colorecta carcinomatosis treated with surgery and perioperative intraperitoneal chemotherapy: Retrospective analysis of 523 patients from a multicentric French study, J Clin Oncol, 2010;28(1):63-8

27. Verwaal VJ, Long-term results of cytoreduction and HIPEC followed by systemic chemotherapy, Cancer J, 2009;15:212-5.

28. Verwaal VJ, van Ruth S, de Bree E, et al., Randomized trial of cytoreduction and hyperthermic intraperitoneal chemotherapy versus systemic chemotherapy and palliative surgery in patients with peritoneal carcinomatosis of colorectal cancer, J Clin Oncol, 2003;21:3737-43.

29. Verwaal VJ, Bruin S, Boot H, et al., 8-year follow up of a randomized trial: Cytoreduction and hyperthermic intraperitoneal chemotherapy versus systemic chemotherapy in patients with peritoneal carcinomatosis of colorectal cancer, Ann Surg Oncol, 2008;15:2426-32.

30. Ryan DP, Cytoreductive surgery and hyperthermic intraperitoneal chemotherapy: History repeating itself or a new standard?, Am Soc Clin Oncol, 2011.

31. Franko J, Ibrahim Z, Gusani NJ, et al., Cytoreductive surgery and hyperthermic intraperitoneal chemotherapy versus systemic chemotherapy alone for colorectal peritoneal carcinomatosis, Cancer, 2010;116(16):3756-62.

32. Sugarbaker $\mathrm{PH}$, Achieving long-term survival with cytoreductive surgery and perioperative chemotherapy to peritoneal surfaces for metastatic colon cancer, Am Soc Clin Oncol, 2011.

33. Pihl E, Hughes ES, McDermott FT, et al., Lung recurrence after curative surgery for colorectal cancer, Dis Colon Rectum, 1987;30(6):417-9

34. Jarabo JR, Fernandez E, Calatayud J, et al., More than one pulmonary resections or combined lung-liver resection in 79 patients with metastatic colorectal carcinoma, J Surg Oncol, 2011;104:781-6.

35. Welter S, Jacobs J, Krbek T, et al., Long-term survival after repeated resection of pulmonary metastases from colorecta cancer, Ann Thorac Surg, 2007;84(1):203-10.

36. De Giacomo T, Rendina EA, Venuta F, et al., Thoracoscopic resection of solitary lung metastases from colorectal cancer is a viable therapeutic option, Chest, 1999;115:1441-3.

37. King J, Glenn D, Clark W, et al., Percutaneous radiofrequency ablation of pulmonary metastases in patients with colorectal cancer, Br J Surg, 2004;91(2):217-23.

38. Elias $D$, Liberale G, Vernerey D, et al., Hepatic and extrahepatic colorectal metastases: When resectable, their localization does not matter, but their total number has a prognostic effect, Ann Surg Oncol, 2005;12(11):900-9.

39. Headrick JR, Miller DL, Nagorney DM, et al., Surgical treatment of hepatic and pulmonary metastases from colon cancer, Ann Thorac Surg, 2001;71(3):975-9.

40. Robinson BJ, Rice TW, Strong SA, et al., Is resection of pulmonary and hepatic metastases warranted in patients with colorectal cancer?, J Thorac Cardiovasc Surg, 1999;117(1):66-75.

41. Elias D, Sideris L, Pocard M, et al., Results of RO resection for colorectal liver metastases associated with extrahepatic disease, Ann Surg Oncol, 2004;11(3):274-80.
42. August DA, Sugarbaker PH, Schneider PD, Lymphatic dissemination of hepatic metastases, Cancer, 1985;55:1490-4.

3. Iwatsuki S, Dvorchik I, Madariaga JR, et al., Hepatic resection for metastatic colorectal adenocarcinoma: a proposal of a prognostic scoring system, J Am Coll Surg, 1999;189:291-9.

44. Eveno C, Goere D, Dartigues P, et al., Ovarian metastasis is associated with retroperitoneal lymph node relapses in women treated for colorectal peritoneal carcinomatosis, Ann Surg Oncol, 2013;20:491-6.

45. Evers DJ, Verwaal VJ, Indication for oophorectomy during cytoreduction for intraperitoneal metastatic spread of colorectal or appendiceal origin, Br J Surg, 2011;98:287-92.

46. Okumura S, Kondo H, Tsuboi M, et al., Pulmonary resection for metastatic colorectal cancer: experiences with 159 patients, I Thorac Cardiovasc Surg, 1996;112(4):867-74.

47. Yedibela S, Klein P, Feuchter K, et al., Surgical management of pulmonary metastases from colorectal cancer in 153 patients, Ann Surg Oncol, 2006;13(11):1538-44.

48. Inoue $\mathrm{M}$, Kotake $\mathrm{Y}$, Nakagawa $\mathrm{K}$, et al., Surgery for pulmonary metastases from colorectal carcinoma, Ann Thorac Surg, 2000;70(2):380-3.

49. Sauer R, Becker H, Hohenberger W, et al., Preoperative versus postoperative chemoradiotherapy for rectal cancer, N Eng/ Med, 2004;351:1731-40

50. Verrees JF, Fernandez-Trigo V, Sugarbaker PH, Rectal cance recurrence after prior resection and radiation therapy: Palliation following additional surgery, Int I Colorectal Dis, 1996;11(5):211-6.

51. Mukherjee A. Total pelvic exenteration for advanced rectal cancer, S D J Med, 1999:52:153-6.

52. Haddock MG, Nelson $\mathrm{H}$, Donohue JH, et al., Intraoperative electron radiotherapy as a component of salvage therapy for patients with colorectal cancer and advanced nodal metastases, Int J Radiat Oncol Biol Phys, 2003;56:966-73.

53. Sugarbaker PH, Cytoreductive surgery plus hyperthermic perioperative chemotherapy for selected patients with peritoneal metastases from colorectal cancer: A new standard of care or an experimental approach?, Gastroenterol Res Pract, 2012;13(8):e362-9.

54. Sugarbaker $\mathrm{PH}$, Second-look surgery for colorectal cancer: Revised selection factors and new treatment options for greater success, Int I Surg Oncol, 2011:2011:015078. 